\title{
Aptitude physique versus adiposité : impacts cardio-métaboliques respectifs chez l'enfant/adolescent et chez la personne âgée
}

\author{
Fitness versus fatness: Respective cardiometabolic impacts \\ in children/adolescents and in elderly people
}

\author{
N. Esser, N. Paquot, A.J. Scheen \\ Université de Liège, Service \\ de diabétologie, nutrition et maladies \\ métaboliques, Département de médecine, \\ $\mathrm{CHU}$, Sart Tilman, Liège, Belgique.
}

\section{Correspondance :}

\section{André Scheen}

Département de médecine

CHU Sart Tilman (B35)

4000 Liège

Belgique

andre.scheen@chu.ulg.ac.be

\begin{abstract}
Résumé
Le sujet adulte d'âge moyen en surpoids ou obèse est caractérisé par une adiposité exagérée, généralement combinée à une aptitude physique cardio-respiratoire déficiente. La pratique régulière d'une activité physique d'endurance améliore le profil de risque cardio-métabolique dans cette tranche d'âge. Le manque d'activité physique chez les adolescents contribue à augmenter leur masse grasse et à induire des anomalies métaboliques, tandis que la sédentarité marquée des sujets âgés peut conduire à un excès de graisse combiné à une fonte musculaire (obésité sarcopénique). Dans ces deux tranches d'âge, les effets néfastes d'un excès de masse grasse (fatness) pourraient être contrecarrés, voire annulés, par la pratique régulière d'exercices musculaires conduisant à une meilleure aptitude physique (fitness). Cet article décrit les relations entre fitness et fatness, et les impacts cardio-métaboliques respectifs de ces deux composantes, d'une part, dans la population jeune $(<20$ ans), d'autre part dans la population âgée (> 60 ans).
\end{abstract}

Mots-clés : Adolescent - aptitude physique - enfant - exercice - obésité - sujet âgé - masse grasse - syndrome métabolique - vieillissement.

\section{Summary}

Middle-aged adult individuals with overweight or obesity are characterized by excessive adiposity, generally combined with poor cardiorespiratory fitness. Endurance exercise training improves cardiometabolic risk profile in such age range. Lack of physical activity among adolescents leads to increased fat mass and development of metabolic abnormalities whereas sedentarity in elderly people may lead to excessive adiposity contrasting with decreased muscular mass (sarcopenic obesity). In both young and elderly people, deleterious cardiometabolic effects of excessive fat mass ("fatness") might be counterbalanced by regular physical activity leading to better cardiorespiratory fitness ("fitness"). The present article analyzes the relationships between "fitness" and "fatness", and the respective cardiometabolic consequences of these two components, in the young population ( $<20$ years), on the one hand, and in the elderly population (> 60 years), on the other hand.

Key-words: Adolescent - fitness - child - exercise - obesity - elderly - fatness - metabolic syndrome - aging. 


\section{Introduction}

L'obésité, en particulier abdominale, s'accompagne souvent de répercussions métaboliques importantes et de complications cardiovasculaires [1, 2], alors que la pratique régulière d'une activité physique, conduisant à une bonne aptitude physique cardio-respiratoire, est généralement associée à une diminution des facteurs de risques [3, 4]. Nous avons analysé, dans un article précédent, les mécanismes physiopathologiques et les interrelations entre fatness (adiposité), fitness (aptitude physique cardio-respiratoire) et la pratique régulière d'exercice musculaire (activité physique). Nous avons ensuite décrit les observations rapportées chez le sujet adulte non diabétique et conclu qu'une bonne aptitude physique, dans cette tranche d'âge, est capable d'atténuer les conséquences cardio-métaboliques liées à un excès d'adiposité [5].

Le but du présent travail est d'analyser les répercussions cardio-métaboliques respectives de l'obésité et de l'aptitude physique dans deux groupes de sujets particulièrement intéressants, situés aux deux extrémités de la vie, à savoir les enfants/adolescents ( $<20$ ans) et les sujets plus âgés (> 60 ans). En effet, dans ces catégories d'âge, il apparaît de plus en plus que la pratique régulière d'une activité physique est essentielle, d'une part, pour prévenir le syndrome métabolique chez l'enfant/adolescent en surpoids [6], d'autre part, pour prévenir l'obésité sarcopénique chez la personne âgée [7] (tableau I). Nous analyserons les impacts respectifs de la fatness et de la fitness chez les personnes avec une diminution de la tolérance au glucose et chez les patients diabétiques de type 2 dans un autre article [8].

\section{Études chez l'enfant et adolescent}

Au vu de l'épidémie d'obésité chez les sujets jeunes, il est crucial de mettre sur pied des stratégies de prévention et de traitement dans cette catégorie d'âge spécifique. Celles-ci doivent se baser sur des modifications de comportement visant à améliorer les habitudes alimentaires et à promouvoir la pratique régulière d'une activité physique [9]. Une des conséquences de l'obésité en pédiatrie est l'augmentation de l'incidence du syndrome métabolique et, plus rarement, du diabète de type 2 (DT2) [10]. La pratique régulière d'exercice physique joue un rôle clé dans la réduction de la masse grasse et des marqueurs de résistance à l'insuline, améliore la condition physique cardio-respiratoire (cardiorespiratory fitness) [11, 12] et atténue l'émergence de syndrome métabolique chez les enfants et les adolescents [7].

Relations entre activité physique, aptitude physique et adiposité

La sédentarité de plus en plus marquée des enfants/adolescents contribue à augmenter leur fatness et à diminuer leur fitness [13]. Dans la European youth heart study, chez 780 enfants âgés de 9-10 ans, une activité physique de forte inten-

Tableau I : Effets cardio-métaboliques favorables procurés par une bonne aptitude physique cardio-respiratoire (fitness) chez les enfants/adolescents et chez les personnes âgées présentant une surcharge pondérale ou une obésité (fatness).

\begin{tabular}{lcc}
\hline Paramètres & Enfants/adolescents & Personnes âgées \\
\hline Adiposité viscérale & $\downarrow$ & $\downarrow$ \\
\hline Masse musculaire & $\uparrow$ & $\uparrow$ \\
\hline Obésité sarcopénique & $\mathrm{n} / \mathrm{a}$ & $\downarrow$ \\
\hline Insulinorésistance musculaire & $\downarrow$ & $\downarrow$ \\
\hline Inflammation silencieuse & $\downarrow$ & $\downarrow$ \\
Syndrome métabolique & $\downarrow$ & $\downarrow$ \\
Risque de diabète de type & $\downarrow$ & $\downarrow$ \\
Hypertension artérielle & $\downarrow$ & $\downarrow$ \\
Mortalité cardiovasculaire & $\mathrm{n} / \mathrm{a}$ & $\downarrow$ \\
Mortalité globale & $\mathrm{n} / \mathrm{a}$ & $\downarrow$ \\
\hline
\end{tabular}

n/a : non applicable.

sité s'avère plus efficace pour prévenir l'obésité et l'adiposité qu'une activité physique plus modérée, alors qu'une activité physique de volume suffisant, bien que d'intensité plus modérée, serait suffisante pour déjà améliorer les facteurs de risque métaboliques [11]. Plusieurs études se sont intéressées à analyser l'influence du nombre d'heures passées devant un écran de télévision ou d'ordinateurs, d'une part, et le degré d'adiposité et le niveau d'aptitude physique des enfants/adolescents, d'autre part [14, 15]. Déjà chez le jeune enfant ( $<6$ ans), il existe une relation entre le temps passé devant la télévision et le degré d'adiposité ou le faible niveau d'activité physique; cependant, la relation entre télévision et adiposité ne semble pas dépendre principalement de la réduction de l'activité physique, mais plutôt, chez ces jeunes enfants, d'une composante de prise alimentaire supplémentaire devant la télévision [14]. Dans une étude longitudinale de 3 années visant à augmenter le niveau d'activité physique et à diminuer le temps d'inactivité passé devant un écran chez des adolescents, âgés de 11 à 19 ans, les changements dans l'aptitude physique sont associés aux modifications de l'indice de masse corporelle (IMC), mais davantage encore aux incréments objectivés dans le niveau d'activité physique [15].

Dans une large population de 4072 enfants, âgés de 9 à 15 ans, recrutés dans différents pays européens, une relation curvilinéaire a été trouvée entre, d'un côté, le degré d'aptitude physique et, de l'autre côté, le tour de taille, la somme des plis adipeux cutanés et la pression artérielle systolique [16]. Les différences étaient plus marquées entre les niveaux d'aptitude physique bas et modérés qu'entre les niveaux modérés et élevés. Cette observation doit encourager à motiver les enfants/adolescents à avoir au moins une activité physique modérée.

Dans une étude grecque, analysant 1362 garçons et filles âgés de 6 à 13 ans, il apparaît que les enfants avec la meilleure aptitude physique sont caractérisés par une moindre adiposité totale et viscérale [17]. Dans une autre étude anglaise, le niveau d'aptitude physique, déterminé par la consommation maximale d'oxygène $\left(\mathrm{VO}_{2} \mathrm{max}\right)$, est 
inversement corrélé à la masse de tissu adipeux viscéral estimée par un examen de tomodensitométrie abdominale dans une population d'enfants âgés de 13 à 14 ans, aussi bien chez les filles que chez les garçons [18]. Enfin, un troisième travail démontre qu'après ajustement pour la masse grasse totale, une plus grande activité physique est associée avec une réduction du tissu adipeux viscéral, mais pas du tissu adipeux sous-cutané, chez des enfants âgés de 8 ans en moyenne [19]. Dans l'ensemble, ces observations confirment donc la forte relation entre un faible niveau d'aptitude physique, le plus souvent lié à une sédentarité exagérée, et un excès de tissu adipeux viscéral chez les enfants/adolescents, comme cela a été démontré amplement dans la population adulte [1, 2].

\section{Impacts sur la sensibilité à l'insuline}

Plusieurs études ont démontré, chez les enfants/adolescents, que la sensibilité à l'insuline est dépendante à la fois du pourcentage de masse grasse (fatness) et du niveau de forme physique (cardiorespiratory fitness) [20]. L'European youth heart study, réalisée sur 873 enfants (moyenne d'âge : 9,4 ans), en Estonie et en Suède, a montré que les marqueurs de résistance à l'insuline sont inversement corrélés au niveau d'aptitude physique. Dans les deux sexes, il existe une relation inverse significative entre le niveau d'activité physique et le degré d'adiposité. Cependant, lorsqu'on analyse la relation entre l'aptitude physique cardiorespiratoire et la sensibilité à l'insuline (évaluée sur la base des concentrations plasmatiques à jeun de glucose et d'insuline, avec la technique HOMA ou Homesostasis model assessment) selon le niveau d'adiposité, les enfants « gras » présentent une grande variation de sensibilité à l'insuline, s'expliquant par les niveaux différents d'aptitude physique. Ainsi, dans cette population, les conséquences délétères provoquées par un haut degré d'adiposité peuvent être contrecarrées par des hauts niveaux d'aptitude cardio-respiratoire [21].

Des différences peuvent cependant être observées entre garçons et filles. Ainsi, Gutin et al. [22] ont montré qu'à la fois le pourcentage de graisse corporelle et le niveau d'aptitude physique expliquent les différences significatives de taux d'insulinémie à jeun mesurés chez les garçons. Par contre, chez les filles, le taux d'insuline à jeun est seulement associé de façon significative au pourcentage de graisse corporelle. Ces observations suggèrent que l'impact néfaste d'un haut niveau de graisse et d'un bas niveau d'aptitude physique est plus important chez les garçons que chez les filles. Cette différence peut être expliquée par le fait que les sujets de cette étude sont plus âgés (14-18 ans) et donc plus matures au niveau biologique, ce qui implique le rôle des hormones sexuelles [22]. Cette observation est en concordance avec I'US national health and nutrition examination survey (NHANES), qui a montré que la sensibilité à l'insuline est significativement associée à l'activité physique et au cardiorespiratory fitness chez les garçons, mais pas chez les filles, où elle est plutôt associée, de façon significative, à I'IMC [23]. Les estrogènes semblent, dans une certaine mesure, protéger les filles des conséquences délétères sur la résistance à l'insuline dues à un faible niveau de cardiorespiratory fitness. Quoi qu'il en soit, une étude grecque, réalisée chez des filles âgées en moyenne de 13 ans, en surpoids ou obèses, a montré qu'un entraînement physique aérobie de 12 semaines améliore la sensibilité à l'insuline, sans réduire le poids corporel ni le pourcentage de masse grasse, et sans modifier les concentrations d'adiponectine, ni les taux des marqueurs inflammatoires [24].

Les effets d'un programme structuré de réentraînement physique pendant 8 semaines ont été évalués chez 14 enfants (8 garçons, 6 filles), âgés de 13 ans en moyenne, présentant une obésité et une augmentation de l'insulinémie à jeun, marqueur indirect d'une insulinorésistance. La sensibilité à l'insuline, mesurée précisément par la technique de référence du glucose clamp, a été significativement améliorée, parallèlement à une augmentation de l'aptitude physique, mais de façon indépendante de modifications de la composition corporelle évaluée par absorptiométrie biphotonique [25].
Impacts sur le développement d'un syndrome métabolique

Dans la population des enfants/adolescents, l'association entre les facteurs de risque cardiovasculaire et le niveau d'activité/aptitude physique est relativement modeste lorsqu'on prend chaque facteur de risque de façon isolée, mais elle apparaît de façon beaucoup plus nette lorsqu'on s'intéresse à un ensemble de facteurs de risque comme ceux inclus dans la définition du syndrome métabolique [26]. La relation entre le niveau d'aptitude physique et le risque de développement d'un syndrome métabolique chez les sujets jeunes a été étudiée dans plusieurs études. Elles démontrent toutes qu'une bonne aptitude physique modifie l'influence de l'adiposité sur le risque métabolique.

Les résultats de la Québec family study, réalisée sur 416 garçons et 345 filles âgés de 9 à 18 ans, indiquent que, dans les différentes catégories d'adiposité (séparées par l'IMC), il existe des différences significatives de risque de syndrome métabolique en fonction du niveau d'aptitude physique : les sujets avec le niveau le plus faible ont le risque le plus élevé de syndrome métabolique [27]. Dans l'étude américaine Physical activity across the curriculum, réalisée sur 375 enfants âgés de 7 à 9 ans, le risque métabolique est significativement plus bas chez les enfants de poids normal et obèses avec un niveau de fitness élevé par rapport aux enfants avec un même IMC, mais un niveau de fitness plus faible [6]. De plus, dans une autre analyse de cette cohorte, il apparaît que l'IMC et le niveau d'aptitude physique sont tous les deux associés au score de syndrome métabolique. Cependant, les enfants avec un surpoids ou une obésité, mais avec un niveau élevé d'aptitude cardiorespiratoire, ont un score de syndrome métabolique plus bas que les enfants de poids normal ayant un faible niveau d'aptitude physique [28]. Dans une autre étude américaine récente, portant sur 1247 adolescents âgés de 12 à 19 ans, les critères de référence d'aptitude cardio-respiratoire (estimation de la $\mathrm{VO}_{2} \max$ à partir de mesures effectuées lors d'un exercice sur tapis roulant) reportés sur un fitnessgram permettent de séparer les sujets avec un meilleur profil de risque 
métabolique de ceux avec un profil particulièrement à risque, en ce compris la pression artérielle systolique, le taux de triglycérides à jeun, le rapport cholestérol total/HDL-cholestérol, l'indice d'insulinorésistance HOMA et la somme des plis cutanés sous-scapulaire et tricipital [29]. Dans une grande étude américaine récente, portant sur 4955 enfants des deux sexes âgés d'environ 12 ans, il a été montré qu'aussi bien l'obésité que le niveau d'aptitude physique influencent les facteurs de risque cardio-métabolique (glucose, insuline, profil lipidique, pression artérielle) [30]. Les résultats de ce travail tendent à montrer qu'à cet âge, les effets de l'obésité sont plus marqués que ceux d'un faible niveau d'aptitude physique, ce qui incite à combattre en priorité l'excès pondéral. Le même type d'analyse a été réalisé dans une population scolaire américaine un peu plus âgée (564 sujets, 19 ans en moyenne) et démontre un effet combiné à la fois de la fatness et de la fitness sur les facteurs de risque cardio-métabolique [31].

Dans la European youth heart study, réalisée chez 1769 enfants, le niveau d'activité physique, le degré d'apti- tude physique, l'adiposité estimée par la mesure des plis cutanés et le tour de taille sont tous associés, de façon indépendante, avec un cluster de facteurs de risque cardiovasculaire, dont l'indice d'insulinorésistance HOMA et plusieurs marqueurs du syndrome métabolique [32]. Par ailleurs, dans une sous-analyse de cette étude réalisée chez 590 enfants âgés de 9-10 ans et chez 535 adolescents âgés de 15-16 ans, il apparaît que le niveau d'aptitude physique (fitness) joue un rôle plus important que le niveau d'activité physique proprement dit sur les divers marqueurs de risque cardiométabolique [33]. Cependant, le niveau d'activité physique joue un rôle plus important à 15-16 ans qu'à 9-10 ans, suggérant que les facteurs modifiables du style de vie prennent plus d'importance avec l'âge. Par ailleurs, une autre analyse de la même cohorte démontre que l'adiposité (fatness) joue un rôle pivot dans l'association entre l'aptitude physique (fitness) et les marqueurs de risque métabolique [34]. Ces résultats ont été confirmés dans une étude suisse, chez 502 enfants âgés de 6 à 12 ans, dans laquelle une faible activité physique et

\section{Les points essentiels}

- La pratique régulière d'une activité physique ainsi qu'un bon niveau d'aptitude cardiorespiratoire (fitness) atténuent les répercussions cardio-métaboliques de l'obésité à tous les âges de la vie, y compris chez les enfants/adolescents $(<20$ ans) et les sujets âgés de plus de 60 ans.

- Chez les enfants/adolescents, il existe une remarquable relation entre un faible niveau d'aptitude physique, le plus souvent lié à une sédentarité exagérée, et un excès pondéral avec accentuation du tissu adipeux viscéral.

-Dans cette population, la pratique régulière d'une activité physique, outre qu'elle contribue à enrayer la progression de l'obésité, améliore les marqueurs de sensibilité à l'insuline et joue un rôle important pour prévenir le syndrome métabolique, même en cas de surpoids, voire d'obésité.

- Chez le sujet âgé de plus de 60 ans, la pratique régulière d'une activité physique permet de prévenir l'obésité sarcopénique, ce qui permet d'améliorer une série de facteurs de risque autres que cardiovasculaires et d'améliorer ainsi la qualité de vie.

- Dans cette tranche d'âge, une bonne aptitude physique contribue à corriger les facteurs de risque cardio-métabolique, dont l'adiposité abdominale, l'insulinorésistance, la diminution de la tolérance au glucose, l'hypertension artérielle et les marqueurs d'inflammation silencieuse.

- Chez le sujet âgé, la pratique régulière d'une activité physique conduisant à une meilleure aptitude cardio-respiratoire a apporté la preuve de sa capacité à réduire l'incidence des événements cardiovasculaires et d'améliorer la survie globale, même en présence d'une augmentation de l'indice de masse corporelle ou du tour de taille. un niveau bas d'aptitude physique sont associés de façon indépendante avec les marqueurs du risque métabolique; cette étude démontre, en outre, que c'est aussi le cas d'un environnement urbain, plus à risque qu'un environnement rural sur le plan cardio-métabolique [35].

Ainsi, les mesures de prévention d'un hyperinsulinisme, marqueur d'une insulinorésistance, et des anomalies métaboliques consécutives, en ce compris l'augmentation de pression artérielle, chez les sujets jeunes doivent se focaliser, non seulement sur la réduction de la graisse, mais aussi sur l'amélioration du niveau d'aptitude physique cardiorespiratoire $[9,21,36]$.

\section{Études chez la personne âgée}

L'étude des relations entre fitness et fatness prend une orientation différente dans la population âgée, par comparaison au groupe des sujets adultes âgés de 20 à 60 ans [5] ou plus encore à celui des enfants/adolescents, notamment en termes de pronostic et d'objectifs thérapeutiques. Ainsi, alors que chez les enfants et adolescents, l'objectif est de limiter le développement du syndrome métabolique et la survenue ultérieure de maladies (dont le DT2) [6], chez la personne âgée, le but principal est d'améliorer la qualité de la vie, mais aussi de prolonger la survie [7].

\section{Notion d'obésité sarcopénique}

Au cours des dernières années est apparu dans la littérature un nouveau concept, avec le terme "obésité sarcopénique »[37, 38]. Ce type d'obésité concerne plus spécifiquement le sujet âgé chez lequel, d'une façon générale, la notion de diminution de la masse musculaire (sarcopénie) est bien connue. Cette sarcopénie relative n'épargne pas le sujet âgé obèse [38] et contribue à aggraver le syndrome cardio-métabolique [37]. Cette diminution de la masse musculaire liée à l'âge dépend de facteurs intrinsèques (programmation génétique, diminution de la sécrétion d'hormone de croissance....) et extrinsèques (alimentation...), et la diminution de l'activité physique y joue sans doute un rôle 
important. Dans ces conditions, on peut faire l'hypothèse qu'un entraînement physique régulier chez la personne âgée devrait contribuer à réduire ce risque d'obésité sarcopénique et ses conséquences délétères pour la santé. Les relations entre obésité sarcopénique et activité physique ne sont cependant pas simples, comme démontré récemment dans une étude canadienne, réalisée chez 904 sujets, âgés de 68 à 82 ans, et en bonne santé [39]. Dans ce travail, les sujets avec obésité sarcopénique n'ont pas de diminution significative de l'activité physique par rapport aux sujets avec obésité mais sans sarcopénie ; ces résultats suggèrent que l'obésité est plus directement associée à la diminution de l'activité physique que la sarcopénie proprement dite.

Rappelons, par ailleurs, que la pratique régulière d'une activité physique par le sujet âgé exerce bien d'autres effets bénéfiques que simplement réduire le risque cardio-métabolique. Ainsi, par exemple, une amélioration du métabolisme osseux, avec une diminution du risque d'ostéoporose, une meilleure stabilité posturale, avec réduction du risque de chute, et une amélioration de la confiance en soi, avec répercussions psychologiques positives et diminution des symptômes dépressifs, ont été rapportées dans la population plus âgée [40].

\section{Impacts sur les facteurs} de risque cardio-métabolique

Le maintien d'une bonne condition physique par la pratique régulière d'un exercice musculaire est donc très important chez les personnes âgées, par exemple au-delà de 60 ans [7]. L'augmentation de la masse (et de la force) musculaire est un but important chez les sujets âgés, même si l'IMC reste inchangé. En effet, le muscle strié squelettique est le plus grand réservoir de glycogène et il est évident que l'augmentation de la masse musculaire, secondaire à la pratique régulière d'une activité physique, joue un rôle favorable sur le contrôle glycémique, en complément de la réduction de la masse grasse [41]. L'étude canadienne, réalisée sur un échantillon de 127 femmes en surpoids ou obèses ménopausées, a montré une relation hautement significative entre la sensibilité à l'insuline et la capacité physique cardio-respiratoire [42].

L'exercice physique améliore de nombreux facteurs qui ont une influence sur la pression artérielle systolique (PAS), paramètre fréquemment élevé dans la population âgée et considéré comme un facteur de risque indépendant de mortalité cardiovasculaire. En effet, on observe une réduction de la PAS après entraînement physique chez les personnes âgées avec hypertension artérielle légère. De plus, les sujets âgés entraînés présentent, de façon indépendante, un tour de taille diminué (marqueur de l'obésité abdominale) et un niveau d'aptitude physique augmenté [43]. Dans une étude réalisée chez 115 sujets, âgés de 55 à 75 ans, bien que la pratique régulière d'exercice améliore le niveau d'aptitude physique, il est apparu que c'est davantage la réduction de l'adiposité totale et abdominale qui est plus fortement associée à une réduction des facteurs de risque cardiovasculaire, y compris ceux du syndrome métabolique, et du DT2 [44].

Enfin, des facteurs génétiques peuvent aussi influencer les effets d'un programme d'entraînement physique sur I'IMC, l'adiposité, l'aptitude physique et les marqueurs de risque métabolique, même chez le sujet âgé. Ainsi, à titre d'exemple, une étude récente a montré, chez des sujets caucasiens âgés, une influence significative d'un polymorphisme du gène codant pour la périlipine, une protéine qui contrôle la lipolyse intracellulaire adipocytaire, en ce qui concerne les effets d'un entraînement musculaire sur le poids corporel, les marqueurs d'adiposité (fatness) et d'aptitude physique (fitness) ainsi que sur divers marqueurs de risque métabolique (dont la diminution de la tolérance au glucose, l'hyperinsulinémie et le taux de HDL-cholestérol abaissé) [45]. Dès lors, les interrelations " gènes-environnement » méritent sans doute d'être mieux étudiées également dans ce domaine particulier.

\section{Impacts sur les marqueurs inflammatoires}

L'obésité, en particulier, celle à distribution abdominale, est associée avec un état d'inflammation silencieuse [1, 2]. Par ailleurs, un haut niveau d'activité physique et d'aptitude physique est associé à une réduction des taux de C-réactive protéine hautement sensible (hsCRP) [46]. L'entraînement physique induit une réduction des concentrations de hsCRP, et cet effet s'avère indépendant des taux de base, de la composition corporelle et de la perte pondérale.

Parmi un nombre élevé de 3075 personnes âgées de 70 à 79 ans, un niveau d'activité physique élevé a été trouvé associé à des taux plus bas des marqueurs inflammatoires, comme les concentrations de hsCRP, de TNF- $\alpha$ (Tumor necrosis factor-alpha) et d'IL-6 (interleukine-6) [47]. Cette association est atténuée, mais persiste néanmoins après ajustement pour le degré d'obésité. Dans une autre étude relative à la population âgée, il a été suggéré qu'une amélioration du tonus parasympathique, grâce à un entraînement physique régulier, pourrait contribuer aux effets anti-inflammatoires de l'exercice, indépendamment du niveau d'aptitude physique ou du degré d'obésité [48].

\section{Impacts sur les complications cardiovasculaires et la mortalité}

Lors d'un suivi prospectif de 8 ans de 3366 sujets canadiens âgés d'au moins 65 ans, indemnes de pathologie cardiovasculaire, les influences respectives de l'excès de masse grasse (IMC, tour de taille, bioimpédance) et de la sarcopénie (déficit de masse et force musculaires) ont été analysées en ce qui concerne l'incidence des événements cardiovasculaires [49]. II apparaît que l'obésité ou la sarcopénie n'est pas suffisante, prise isolément, pour augmenter le risque cardiovasculaire, mais qu'une combinaison des deux conditions accroît le risque, quoique de façon relativement modérée. Les données suggèrent aussi que la force musculaire paraît jouer un plus grand rôle protecteur que la masse musculaire proprement dite.

L'obésité ne joue pas nécessairement le même rôle délétère chez les sujets âgés que chez les sujets plus jeunes. Ainsi, dans l'étude américaine Veterans 
exercise testing study, analysant la mortalité lors d'un suivi de près de 7 ans chez 681 hommes, âgés d'au moins 65 ans, il a été rapporté qu'un faible poids corporel et un faible niveau d'aptitude physique sont deux facteurs indépendants de mortalité [50]. De façon intéressante, dans cette cohorte, la mortalité la plus basse a été observée dans le groupe d'hommes obèses avec un haut niveau de fitness. Néanmoins, l'augmentation du tour de taille reste grevée d'une surmortalité dans cette tranche d'âge, comme l'a montré l'Aerobics center longitudinal study, chez 2603 individus des deux sexes âgés de plus de 60 ans [7]. Dans cette étude, le niveau d'aptitude physique est fortement corrélé, de façon inverse, avec la mortalité et ce, indépendamment du tabagisme, du niveau de santé de base, du tour de taille, de l'IMC et du taux de graisse corporelle. Plus intéressant encore, l'ajustement pour l'aptitude physique fait complètement disparaître la relation entre mortalité et tour de taille dans cette population âgée.

\section{Conflits d'intérêt}

Les auteurs n'ont aucun conflit d'intérêt en relation avec le contenu de cet article.

\section{Références}

[1] Després JP, Lemieux I. Abdominal obesity and metabolic syndrome. Nature 2006;444:881-7.

[2] Van Gaal LF, Mertens IL, De Block CE. Mechanisms linking obesity with cardiovascular disease. Nature 2006;444:875-80.

[3] Blair SN, Morris JN. Healthy hearts and the universal benefits of being physically active: Physical activity and health. Ann Epidemiol 2009;19:253-6.

[4] Fogelholm M. Physical activity, fitness and fatness: Relations to mortality, morbidity and disease risk factors. A systematic review. Obes Rev 2010;11:202-21.

[5] Esser N, Paquot N, Scheen AJ. Aptitude physique versus adiposité : aspects physiopathologiques et impacts cardio-métaboliques chez le sujet adulte non diabétique. Médecine des maladies Métaboliques 2010;4:291-8.

[6] Eisenmann JC. Aerobic fitness, fatness and the metabolic syndrome in children and adolescents. Acta Paediatr 2007;96:1723-9.

[7] Sui X, LaMonte MJ, Laditka JN, et al. Cardiorespiratory fitness and adiposity as mortality predictors in older adults. JAMA 2007;298:2507-16.

[8] Esser N, Paquot N, Scheen AJ. Aptitude physique versus adiposité : impacts métaboliques respectifs chez le sujet avec une diminution de la tolérance au glucose ou un diabète de type 2 . Maladies des maladies Métaboliques 2010;4: soumis.

[9] August GP, Caprio S, Fennoy I, et al. Prevention and treatment of pediatric obesity: An endocrine society clinical practice guideline based on expert opinion. J Clin Endocrinol Metab 2008;93:4576-99.

[10] Steinberger J, Daniels SR, Eckel RH, et al. American Heart Association Atherosclerosis, Hypertension, and Obesity in the Young Committee

\section{Conclusion}

Un bon niveau d'aptitude physique permet de contrecarrer - voire même d'annuler - les conséquences néfastes d'un excès de masse grasse et ce, aux périodes extrêmes de la vie comme chez l'adulte d'âge moyen [5]. Chez les enfants et les adolescents, la pratique régulière d'une activité physique améliore la sensibilité à l'insuline et réduit le risque de développement d'un syndrome métabolique, sans nécessairement passer par une perte importante de poids. Chez les personnes âgées, le maintien d'une bonne condition physique permet de lutter contre l'obésité « sarcopénique » et les complications cardio-métaboliques qui lui sont associées, en particulier l'hypertension artérielle systolique et l'inflammation silencieuse, tout en diminuant le risque d'ostéoporose et de dépression. Dans cette tranche d'âge, une bonne aptitude physique améliore le pronostic cardiovasculaire et réduit la mortalité. Des recommandations doivent donc être données pour la pratique régulière d'une activité physique adaptée chez les sujets plus âgés [51].

Trop souvent, on constate que l'excès pondéral est associé à une moins bonne aptitude physique. Il est donc important d'encourager les personnes en surpoids ou obèses à rester actives. Le maintien d'une activité physique régulière a une place primordiale dans la prévention primaire des pathologies cardio-métaboliques chez l'adolescent, et de l'obésité sarcopénique - capable d'altérer qualité de vie et pronostic vital - chez la personne âgée.

of the Council on Cardiovascular Disease in the Young; Council on Cardiovascular Nursing; and Council on Nutrition, Physical Activity, and Metabolism. Progress and challenges in metabolic syndrome in children and adolescents: A scientific statement from the American Heart Association Atherosclerosis, Hypertension, and Obesity in the Young Committee of the Council on Cardiovascular Disease in the Young; Council on Cardiovascular Nursing; and Council on Nutrition, Physical Activity, and Metabolism. Circulation 2009;119:628-47.

[11] Ruiz JR, Rizzo NS, Hurtig-Wennlöf A, et al. Relations of total physical activity and intensity to fitness and fatness in children: The European Youth Heart Study. Am J Clin Nutr 2006;84:299 303 [Erratum in: Am J Clin Nutr 2009;89:656].

[12] Platat C, Wagner A, Klumpp T, et al. Relationships of physical activity with metabolic syndrome features and low-grade inflammation in adolescents. Diabetologia 2006;49:2078-85.

[13] Olds TS, Ridley K, Tomkinson GR. Declines in aerobic fitness: Are they only due to increasing fatness? Med Sports Sci 2007;50:226-40.

[14] Jackson DM, Djafarian K, Stewart J, et al. Increased television viewing is associated with elevated body fatness but not with lower total energy expenditure in children. Am $\mathrm{J}$ Clin Nutr 2009;89:1031-6.

[15] Aires L, Andersen LB, Mendonça D, et al. A 3-year longitudinal analysis of changes in fitness, physical activity, fatness and screen time. Acta Paediatr 2010;99:140-4 [Erratum in: Acta Paediatr 2010;99:153].

[16] Klasson-Heggebø L, Andersen LB, Wennlöf $\mathrm{AH}$, et al. Graded associations between cardiorespiratory fitness, fatness, and blood pressure in children and adolescents. Br J Sports Med 2006;40:25-9; discussion 25-9.

[17] Nassis GP, Psarra G, Sidossis LS. Central and total adiposity are lower in overweight and obese children with high cardiorespiratory fitness. Eur J Clin Nutr 2005;59:137-41.

[18] Winsley RJ, Amstrong N, Middlebrooke AR, et al. Aerobic fitness and visceral adipose tissue in children. Acta Paediatr 2006;95:1435-8.

[19] Saelens BE, Seeley BE, van Schaick K, et al. Visceral abdominal fat is correlated with whole-body fat and physical activity among 8$\mathrm{y}$-old children at risk of obesity. Am J Clin Nutr 2007;85:46-53.

[20] Krekoukia M, Nassis GP, Psarra G, et al. Elevated total and central adiposity and low physical activity are associated with insulin resistance in children. Metabolism 2007;56:206-13.

[21] Ruiz JR, Rizzo NS, Ortega FB, et al. Markers of insulin resistance are associated with fatness and fitness in school-aged children: The European Young Heart Study. Diabetologia 2007;50:1401-8

[22] Gutin B, Yin Z, Humphries MC, et al. Relations of moderate and vigorous physical activity to fitness and fatness in adolescents. Am J Clin Nutr 2005;81:746-50.

[23] Imperatore G, Cheng YJ, Williams DE, et al. Physical activity, cardiovascular fitness, and insulin sensitivity among U.S. adolescents: The National Health and Nutrition Examination Survey, 1999-2002. Diabetes Care 2006;29:1567-72. 
[24] Nassis GP, Papantakou K, Skenderi K, et al. Aerobic exercise training improves insulin sensitivity without changes in body weight, body fat, adiponectin, and inflammatory markers in overweight and obese girls. Metabolism 2005;54:1472-9.

[25] Bell LM, Watts K, Siafarikas A, et al. Exercise alone reduces insulin resistance in obese children independently of changes in body composition. J Clin Endocrinol Metab 2007;92:4230-5.

[26] Froberg K, Andersen LB. Mini review: Physical activity and fitness and its relations to cardiovascular disease risk factors in children. Int J Obes (Lond) 2005;29(Suppl.2):S34-9.

[27] Eisenmann JC, Katzmarzyk PT, Perusse L, et al. Aerobic fitness, body mass index, and CVD risk factors among adolescents: The Québec family study. Int J Obes (Lond) 2005;29:1077-83.

[28] DuBose KD, Eisenmann JC, Donnelly JE. Aerobic fitness attenuates the metabolic syndrome score in normal-weight, at-risk-foroverweight, and overweight children. Pediatrics 2007;120:e1262-8.

[29] Lobelo F, Pate RR, Dowda M, et al. Validity of cardiorespiratory fitness criterion-referenced standards for adolescents. Med Sci Sports Exerc 2009;41:1222-9.

[30] Jago R, Drews KL, McMurray RG, et al; the HEALTHY Study Group. Fatness, fitness, and cardiometabolic risk factors among sixth grade youth. Med Sci Sports Exerc 2010;42:1502-10

[31] Sacheck JM, Kuder JF, Economos CD. Physical fitness, adiposity, and metabolic risk factors in young college students. Med Sci Sports Exerc 2010;42:1039-44.

[32] Andersen LB, Sardinha LB, Froberg K, et al. Fitness, fatness and clustering of cardiovascular risk factors in children from Denmark, Estonia and Portugal: The European Youth Heart Study. Int $J$ Pediatr Obes 2008;3(Suppl.1):58-66.
[33] Hurtig-Wennlöf A, Ruiz JR, Harro M, et al. Cardiorespiratory fitness relates more strongly than physical activity to cardiovascular disease risk factors in healthy children and adolescents: The European Youth Heart Study. Eur J Cardiovasc Prev Rehabil 2007;14:575-81.

[34] Rizzo NS, Ruiz JR, Hurtig-Wennlöf A, et al. Relationship of physical activity, fitness, and fatness with clustered metabolic risk in children and adolescents: The European youth heart study. J Pediatr 2007;150:388-94 [Erratum in: J Pediatr 2008;153:874].

[35] Kriemler S, Manser-Wenger S, Zahner L, et al. Reduced cardiorespiratory fitness, low physical activity and an urban environment are independently associated with increased cardiovascular risk in children. Diabetologia 2008;51:1408-15.

[36] Weiss R, Raz I. Focus on childhood fitness, not just fatness. Lancet 2006;368:261-2.

[37] Dominquez LJ, Barbagallo M. The cardiometabolic syndrome and sarcopenic obesity in older persons. J Cardiometab Syndr 2007;2:183-9.

[38] Zamboni M, Mazzali G, Fantin F, et al. Sarcopenic obesity: a new category of obesity in the elderly. Nutr Metab Cardiovasc Dis 2008;18:388-95.

[39] Bouchard DR, Dionne IJ, Brochu M. Sarcopenic/obesity and physical capacity in older men and women: data from the Nutrition as a Determinant of Successful Aging (NuAge) - the Quebec Longitudinal Study. Obesity (Silver Spring) 2009;17:2082-8.

[40] American College of Sports Medicine, Chodzko-Zajlo WJ, Proctor DN, et al; American College of Sports Medicine position stand. Exercise and physical activity for older adults. Med Sci Sports Exerc Med 2009;41:1510-30.

[41] Kay SJ, Fiatarone Singh MA. The influence of physical activity on abdominal fat: A systematic review of the literature. Obes Rev 2006;7:183200.
[42] Messier V, Malita FM, Rabasa-Lhoret R, et al. Association of cardiorespiratory fitness with insulin sensitivity in overweight and obese postmenopausal women: A Montreal Ottawa New Emerging Team study. Metabolism 2008;57:1293-8.

[43] Barone BB, Wang NY, Bacher AC, et al. Decreased exercise blood pressure in older adults after exercise training: Contributions of increased fitness and decreased fatness. Br J Sports Med 2009;43:52-6.

[44] Stewart KJ, Bacher AC, Turner K, et al. Exercise and risk factors associated with metabolic syndrome in older adults. Am J Prev Med 2005;28:9-18.

[45] Jenkins NT, McKenzie JA, Damcott CM, et al. Endurance exercise training effects on body fatness, VO2max, HDL-C subfractions, and glucose tolerance are influenced by a PLIN haplotype in older Caucasians. J Appl Physiol 2010;108:498 509.

[46] Plaisance EP, Grandjean PW. Physical activity and high-sensitivity C-reactive protein. Sports Med 2006;36:443-58.

[47] Colbert LH, Visser M, Simonsick EM, et al. Physical activity, exercise, and inflammatory markers in older adults: findings from the Health, Aging and Body Composition Study. J Am Geriatr Soc 2004;52:1098-104.

[48] Vieira VJ, Valentine RJ, McAuley E, et al. Independent relationship between heart rate recovery and C-reactive protein in older adults. J Am Geriatr Soc 2007;55:747-51.

[49] Stephen WC, Janssen I. Sarcopenic-obesity and cardiovascular disease risk in the elderly. $J$ Nutr Health Aging 2009;13:460-6.

[50] McAuley P, Pittsley J, Myers J, et al. Fitness and fatness as mortality predictors in healthy older men: the Veterans exercise testing study. J Gerontol A Biol Sci Med Sci 2009;64:695-9.

[51] Elsawy B, Higgins KE. Physical activity guidelines for older adults. Am Fam Physician 2010;81:55-9. 